\title{
Correspondence
}

\section{Stimulus dosing and titration in electroconvulsive therapy}

Sir: I understand that the manufacturer of the Neurotronic Therapy System recently produced two new versions of their ECT device, the SLE NTS-C and the SLE NTS-R. Given the frustrating experiences some of my colleagues and I have had with the original model, I was interested to read the article on the pitfalls of dose titration in electroconvulsive therapy by Byrne et al (Psychiatric Bulletin, 1995, 19. 207-208).

Although not explicitly stated, it appears that the recommended dose titration schedule (p. 207) refers to use of the new SLE NTS-C model since neither the original version nor the new SLE NTS-R have a pulse width setting of 1 millisecond. In fact, according to the manufacturer's specifications the SLE NTS-R, ostensibly produced for research applications, does not differ substantially from the earlier device, apart from an increase in the maximum output potential from $\sim 200$ Volt to $\sim 400$ Volt - a feature which it shares with the SLE NTS-C. The reason for this change is not clear but may have been prompted by the difficulties some clinicians experienced inducing adequate seizures with the original model. My own enquiries to the manufacturer of the Neurotronic Therapy System a few years ago regarding dose titration guidelines were to no avail. Curiously, the SLE NTS-R has retained frequency $(150 \mathrm{Herz})$, pulse width $(2$ and 2.5 milliseconds) and current mode (monophasic) settings which may not be therapeutically efficient (Swartz \& Abrams, 1993; Sackeim et al, 1994).

Constant current stimulation is generally accepted as the most physiological method for inducing neuronal depolarisation and inducing seizures (Abrams, 1992). Although the various ECT devices currently available differ widely, most, unlike the new SLE NTS-C model, are designed to give a fixed constant output, e.g. the Tymatron DG (900 milliAmpere), the MECTA SR-2 and JR-2 (800 milliAmpere) and the ECTONUS Series 5 (750 milliAmpere). Stimulus dose charges are titrated in increments of milliCoulomb. Byrne and colleagues correctly point out that while summary measures of stimulus intensity may be constant despite radically different configurations of stimulus parameters, it should not be assumed that the seizure-eliciting potential of different stimulus packages is identical. Nevertheless, their apparent advocacy of the fixed parameters of the SLE NTS-C (p. 207) fails to take into account that manipulating the stimulus train duration may be an efficient means of adjusting the stimulus dose relative to increasing current intensity in selected patients who would otherwise fail to attain a seizure (Swartz \& Larson, 1989; Swartz \& Abrams, 1993; Sackeim et al, 1994).

Finally, the maximum stimulus setting of the SLE NTS-C, i.e. 1200 milliAmpere (or 720 milliCoulomb, according to my calculations), seems worryingly low. Given my own experience with the Neurotronic Therapy System, I fear clinicians, especially psychogeriatricians, may have difficulties inducing or maintaining adequate seizures in their patients. During a course of ECT it is often, particularly with elderly male patients, necessary to increase the stimulus to maintain adequate seizure induction and duration (Sackeim et al, 1991; Abrams, 1992). Again, in my experience, the recommendation of Byrne et al (p. 208) of an increase in stimulus of 10 to 35 milliAmpere (or 6 to 21 milliCoulomb) at each treatment session seems too conservative and may be insufficient to optimise the therapy of individual patients.

ABRams, R. (1992) Electroconvulsive Therapy. (2nd Edn). New York: Oxford Untversity Press.

SaCkeim, H. A., Devanand, D. P. \& Prudic, J. (1991) Stimulus intensity, seizure threshold, and seizure duration. Psychiatric Clinics of North America, 14, 803-843.

-. LONG, J., LUBER, B., et al (1994) Physical properties and quantification of the ECT stimulus. Convulsive Therapy. 10, 93-123.

SWARTZ, C. M. \& LARSON, G. (1989) ECT stimulus duration and its efficacy. Annals of Clinical Psychiatry. 1. 147-152.

- \& ABRAMS, R. (1993) ECT Instruction Manual (4th Edn). Lake Bluff: Somatics, Inc.

KAREL DE PAUW

St James's University Hospital, Beckett Street, Leeds LS9 7TF 